\begin{tabular}{ll}
\hline Frontiers in Heat and Mass Transfer \\
Available at www.ThermalFluidsCentral.org
\end{tabular}

\title{
TRANSIENT FREE CONVECTION MHD FLOW PAST A VERTICAL PLATE WITH EXPONENTIALLY DECAYING WALL TEMPERATURE AND RADIATION
}

\author{
Rudra Kanta Deka ${ }^{\mathrm{a}}$, Ashish Paul ${ }^{\mathrm{b}, *}$, Nityajyoti Kalita ${ }^{\mathrm{c}}$ \\ ${ }^{a}$ Department of Mathematics, Gauhati University, Guwahati-781014, India \\ ${ }^{b}$ Department of Mathematics, Cotton College State University, Guwahati-781001, India \\ ${ }^{c}$ Academic Officer, Board of Secondary Education, Assam, Guwahati-781021, India
}

\begin{abstract}
A theoretical study of thermal radiation effects on unsteady MHD natural convection flow of an electrically conducting fluid past a vertical plate with variable temperature is considered. It is supposed that the temperature of the plate decays exponentially with time. Exact solutions to the nondimensionalised coupled linear partial differential equations representing the flow problem are obtained using Laplace transform technique. Effects of different physical parameters involved in the temperature and velocity profiles are investigated, shown graphically and discussed. Skin friction and Nusselt number are also derived and their variations with respect to the parameters are investigated.
\end{abstract}

Key words: Vertical plate, free convection, radiation, $M H D$ flow.

\section{INTRODUCTION}

Natural convection MHD flow of a viscous incompressible electrically conducting fluid with simultaneous effects of radiation has been drawing considerable attention due to its significant effects on the boundary layer flow control. Numerous studies have been carried out in this field by many researchers under different flow situations. In contrast to the mechanism of heat transfer in fluids, the importance of electromagnetic radiation can never be neglected. Accordingly, in recent years, progress has been considerably made in the study of heat transfer with simultaneous effects of radiation. Radiation and on the optical properties of the emitter, with its internal energy being converted to, is the process of heat propagation by means of electromagnetic waves. Radiative convective flows are encountered in countless industrial and environmental processes, particularly in astrophysical phenomenon and technologies in space dynamics. In space technology applications and at higher operating temperatures, effect of radiations is quite significant. Several investigations have been done which are concerned with radiative MHD flows past flat plate considering different flow situations. Hossain and Takhar (1996) investigated radiation effects on mixed convection along vertical plate with uniform surface temperature. Das et al. (1996) studied radiation effects on flow past impulsively started vertical plate. Later on, Muthucumaraswamy and Kumar (2004) observed the heat and mass transfer effects on moving vertical plate in presence of thermal radiation.

In case of electrically conducting fluid flows, it has been observed that the effect of transversely applied magnetic field plays pronounced role over radiation in the whole flow characteristics. In recent years, more analytical and numerical results on unsteady free convection flow past semi-infinite vertical plate were presented by a good numbers of researchers. Ibrahim et al. (2008) observed the influences of viscous dissipation and radiation on unsteady MHD mixed convection flows of micro-polar fluids, while Mahmoud Mustafa (2007) investigated the flow of fluids with variable viscosity past a moving vertical plate with simultaneous effects of transverse magnetic field and radiation. Ramachandra et al. (2007) studied radiation and mass transfer effects on an unsteady MHD free convective flow past a heated vertical plate in porous medium with viscous dissipation. Studies on flow past plate with variable temperature have been receiving quite remarkable attention by researchers since long back. Temperature variations at the plate may occur with different modes with respect to the source responsible for heating the surface bounding the fluid. Nature of a flow obviously depends on how temperature of the plate varies. Realizing the importance of such flows some researchers had made their investigations on this topic. Das et al. (1999) investigated transient free convection flow past an infinite vertical plate with periodic temperature variation. $\mathrm{Pal}$ and Chatterjee (2010) studied heat and mass transfer in MHD non-Darcian flow of a micro-polar fluid over a stretching sheet embedded in a porous medium with non-uniform heat source and thermal radiation.

In the present study we have studied the radiative heat transfer MHD flow past a vertical plate with exponentially decaying plate temperature. Closed form solutions of the flow problem are obtained by using Laplace transform technique. Nusselt number and skin friction are also derived and the effects of different parameters on the flow characteristics are investigated and presented graphically.

${ }^{*}$ Corresponding author. Email: ashpaul85@gmail.com 


\section{MATHEMATICAL FORMULATION}

Here we have considered a laminar MHD free convection flow of a viscous, incompressible fluid past an infinite vertical plate with thermal radiation. The physical model of the problem is shown in Fig.1. Here the $x^{\prime}$ - axis is taken along the plate in the vertically upward direction and $y^{\prime}$-axis is taken normal to the plate. Initially, at $t^{\prime} \leq 0$, the plate and the fluid is at the same temperature $T_{\infty}^{\prime}$. It is assumed that at time $t^{\prime}>0$ the plate temperature is raised to $T_{\infty}^{\prime}+\left(T_{w}^{\prime}-T_{\infty}^{\prime}\right)+\operatorname{Exp}\left(-c t^{\prime}\right)$. The viscous dissipation is assumed to be negligible in the energy equation as the motion is due to free convection only. Under these assumptions with usual Boussinesq's approximation the governing boundary layer equations are:

$$
\begin{aligned}
& \frac{\partial u^{\prime}}{\partial t^{\prime}}=g \beta\left(T^{\prime}-T_{\infty}^{\prime}\right)+v \frac{\partial^{2} u^{\prime}}{\partial y^{\prime 2}}-\frac{\sigma B_{0}^{2}}{\rho} u^{\prime} \\
& \rho C_{p} \frac{\partial T^{\prime}}{\partial t^{\prime}}=\kappa \frac{\partial^{2} T^{\prime}}{\partial y^{\prime 2}}-\frac{\partial q_{r}}{\partial y^{\prime}}
\end{aligned}
$$

with initial and boundary conditions as:

$$
\begin{aligned}
& t^{\prime} \leq 0: u^{\prime}=0, \quad T^{\prime}=T_{\infty}^{\prime} \\
& \text { for all } y^{\prime} \\
& t^{\prime}>0: u^{\prime}=0, \quad T^{\prime}=T_{\infty}^{\prime}+\left(T_{w}^{\prime}-T_{\infty}^{\prime}\right) e^{-c t^{\prime}} \text { at } y^{\prime}=0 \\
& \& u^{\prime} \rightarrow 0, T^{\prime} \rightarrow T_{\infty}^{\prime} \quad \text { as } y^{\prime} \rightarrow \infty
\end{aligned}
$$

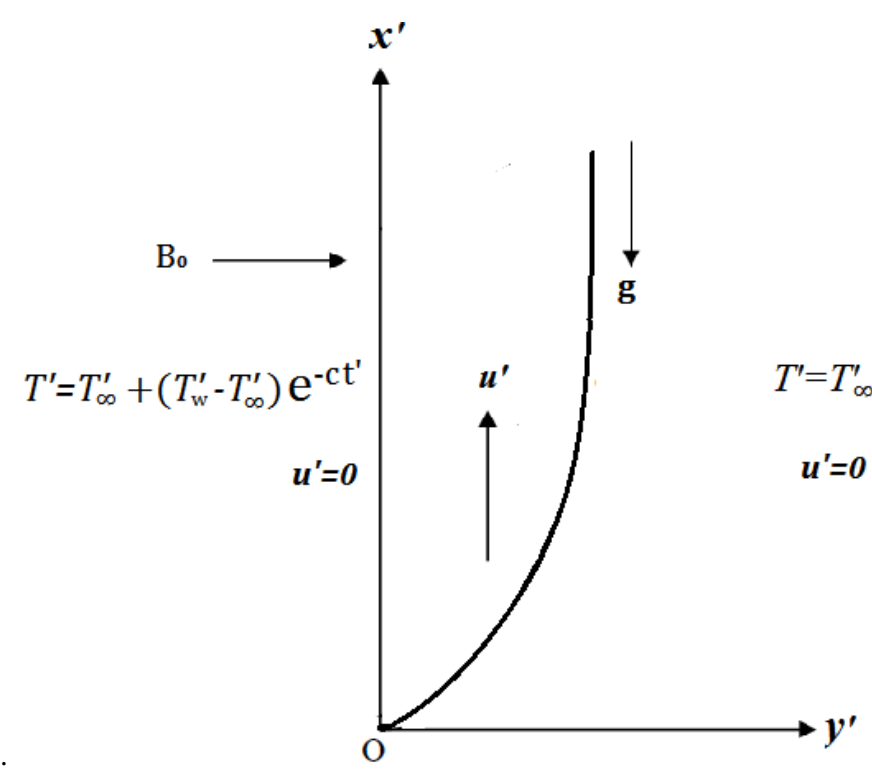

Fig. 1 The physical model and coordinate system.

Here $\mathrm{u}^{\prime}$ is the fluid velocity in $x^{\prime}$ - direction, $\mathrm{T}^{\prime}$ the temperature, $T_{\mathrm{w}}$ the temperature at the plate, $T_{\infty}$ the temperature far away from the plate, $\mathrm{B}_{0}$ the magnetic field strength, $q_{r}$ the radiative flux, $C_{p}$ the specific heat at constant pressure, $\mathrm{t}^{\prime}$ the time, $\mathrm{c}$ is any constant, $\sigma$ the electrical conductivity, $\rho$ the density, $\beta$ the coefficient of volume expansion, $g$ the acceleration due to gravity, $v$ the kinetic viscosity and $\kappa$ the thermal conductivity.
The term $\frac{\partial q_{r}}{\partial y^{\prime}}$ in equation (2) represents the change in the radiative flux with the distance normal to the plate.

For an optically thin gray gas, the local radiant is given by,

$\frac{\partial q_{r}}{\partial y^{\prime}}=-4 a^{*} \sigma^{*}\left(T_{\infty}^{\prime 4}-T^{\prime 4}\right)$

If we assume that the temperature difference within the flow is sufficiently small, so that $T^{\prime 4}$ may be expressed as a linear function of the temperature. This facilitates to expand $T^{\prime 4}$ by Taylor's series about $T_{\infty}^{\prime}$. Neglecting the higher order terms in the expansion, we get

$T^{\prime 4}=4 T_{\infty}^{\prime 3} T^{\prime}-3 T_{\infty}^{\prime 4}$

Using (4) and (5) in (2) we get

$\rho C_{p} \frac{\partial T^{\prime}}{\partial t^{\prime}}=\kappa \frac{\partial^{2} T^{\prime}}{\partial y^{\prime 2}}-16 a^{*} \sigma^{*} T_{\infty}^{\prime 3}\left(T^{\prime}-T_{\infty}^{\prime}\right)$

On introducing the following non dimensional quantities,

$u=\frac{u^{\prime}}{U_{0}}, \quad y=\frac{y^{\prime} U_{0}}{v}, \quad t=\frac{t^{\prime} U_{0}^{2}}{v}, \quad \theta=\frac{T^{\prime}-T_{\infty}^{\prime}}{T_{W}^{\prime}-T_{\infty}^{\prime}}, \quad \operatorname{Pr}=\frac{\mu C_{p}}{\kappa}$, $G=\frac{g \beta v\left(T_{W}^{\prime}-T_{\infty}^{\prime}\right)}{U_{0}^{3}}, F=\frac{16 a^{*} \sigma^{*} T_{\infty}^{\prime 3} v^{2}}{\kappa U_{0}^{2}}$,

$q=\frac{(c v)^{1 / 2}}{U_{0}}, \quad M=\frac{\sigma B_{0}^{2} v}{\rho U_{0}^{2}}$

equations (1) and (6) respectively become,

$\frac{\partial u}{\partial t}=\frac{\partial^{2} u}{\partial y^{2}}+G \theta-M u$

$\operatorname{Pr} \frac{\partial \theta}{\partial t}=\frac{\partial^{2} \theta}{\partial y^{2}}-F \theta$

and the corresponding initial and boundary conditions are,

$$
\left.\begin{array}{rl}
t^{\prime} \leq 0: u=0, & \theta=0 \quad \text { for all } \quad y \\
t^{\prime}>0: & u=0, \quad \theta=e^{-q t} \text { at } y=0 \\
& u \rightarrow 0, \quad \theta \rightarrow 0 \quad \text { as } y \rightarrow \infty
\end{array}\right\}
$$

where $\mathrm{u}, \theta$ and $\mathrm{t}$ represents the dimensionless velocity, temperature and time respectively; $U_{0}$ is the reference velocity, Pr the Prandtl number G is the Grashof number, $\mathrm{F}$ the radiation parameter, $\mathrm{M}$ the Hartmann number and $\mathrm{q}$ the decay parameter. 


\section{ANALYTICAL SOLUTION}

To solve the unsteady linear governing equations (8) and (9) subject to initial and boundary conditions (10), we apply Laplace transform technique. Accordingly we get,

$$
\begin{aligned}
& \frac{d^{2} \bar{u}}{d y^{2}}-(s+M) \bar{u}+G \bar{\theta}=0 \\
& \frac{d^{2} \bar{\theta}}{d y^{2}}-(\operatorname{Pr} s+F) \bar{\theta}=0
\end{aligned}
$$

Solutions of the coupled equations (11) and (12) subject to the Laplace transformations of boundary conditions (10) give the velocity and temperature profiles as [using Hetnarski (1964) and Hetnarski (1975)]

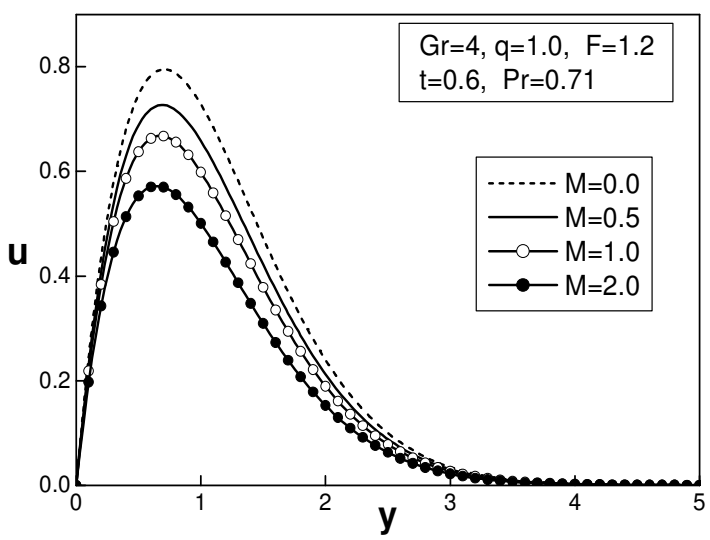

Fig. 2 Magnetic field effect on velocity profile against $y$.

$$
\begin{aligned}
& u=\frac{G r}{2(F-M)}\left[e^{-y \sqrt{M}} \operatorname{erfc}\left(\frac{y}{2 \sqrt{t}}-\sqrt{M t}\right)+e^{y \sqrt{M}} \operatorname{erfc}\left(\frac{y}{2 \sqrt{t}}+\sqrt{M t}\right)\right. \\
& \left.-e^{-y \sqrt{F}} \operatorname{erfc}\left(\frac{y \sqrt{\mathrm{Pr}}}{2 \sqrt{t}}-\sqrt{\frac{F}{\operatorname{Pr}} t}\right)-e^{y \sqrt{F}} \operatorname{erfc}\left(\frac{y \sqrt{\operatorname{Pr}}}{2 \sqrt{t}}+\sqrt{\frac{F}{\operatorname{Pr}} t}\right)\right] \\
& +\frac{G r e^{-q t}}{2(F-M-q(\operatorname{Pr}-1))}\left[e^{-y \sqrt{M-q}} \operatorname{erfc}\left(\frac{y}{2 \sqrt{t}}-\sqrt{(M-q) t}\right)\right. \\
& +e^{y \sqrt{M-q}} \operatorname{erfc}\left(\frac{y}{2 \sqrt{t}}+\sqrt{(M-q) t}\right)-e^{-y \sqrt{F-q \operatorname{Pr}}} \operatorname{erfc}\left(\frac{y \sqrt{\operatorname{Pr}}}{2 \sqrt{t}}-\sqrt{\left(\frac{F}{\operatorname{Pr}}-q\right) t}\right) \\
& \left.-e^{y \sqrt{F-q \operatorname{Pr}}} \operatorname{erfc}\left(\frac{y \sqrt{\operatorname{Pr}}}{2 \sqrt{t}}+\sqrt{\left(\frac{F}{\operatorname{Pr}}-q\right) t}\right)\right] \\
& -\frac{G r}{2}\left\{\frac{1}{F-M}+\frac{1}{F-M-q(\operatorname{Pr}-1)}\right\} e^{-t \frac{F-M}{\operatorname{Pr}-1}}\left[e^{-y \sqrt{\frac{M \operatorname{Pr}-F}{\operatorname{Pr}-1}}}\right. \\
& \operatorname{erfc}\left(\frac{y}{2 \sqrt{t}}-\sqrt{\frac{M \operatorname{Pr}-F}{\operatorname{Pr}-1} t}\right)+e^{y \sqrt{\frac{M \operatorname{Pr}-F}{\operatorname{Pr}-1}}} \operatorname{erfc}\left(\frac{y}{2 \sqrt{t}}+\sqrt{\frac{M \operatorname{Pr}-F}{\operatorname{Pr}-1} t}\right) \\
& -e^{-y \sqrt{\frac{M \operatorname{Pr}-F}{\operatorname{Pr}-1}}} \operatorname{erfc}\left(\frac{y \sqrt{\operatorname{Pr}}}{2 \sqrt{t}}-\sqrt{\frac{M \operatorname{Pr}-F}{(\operatorname{Pr}-1) \operatorname{Pr}} t}\right) \\
& \left.-e^{y \sqrt{\frac{M \operatorname{Pr}-F}{\operatorname{Pr}-1}}} \operatorname{erfc}\left(\frac{y \sqrt{\operatorname{Pr}}}{2 \sqrt{t}}+\sqrt{\frac{M \operatorname{Pr}-F}{(\operatorname{Pr}-1) \operatorname{Pr}}} t\right)\right]
\end{aligned}
$$

$$
\begin{gathered}
\theta=\frac{e^{-q t}}{2}\left\{e^{-y \sqrt{F-q \operatorname{Pr}}} \operatorname{erfc}\left(\frac{y \sqrt{\operatorname{Pr}}}{2 \sqrt{t}}-\sqrt{(F-q \operatorname{Pr}) t}\right)\right. \\
\left.+e^{y \sqrt{(F-q \operatorname{Pr}) \operatorname{Pr}}} \operatorname{erfc}\left(\frac{y \sqrt{\operatorname{Pr}}}{2 \sqrt{t}}+\sqrt{(F-q \operatorname{Pr}) t}\right)\right\} \\
+\frac{1}{2}\left\{e^{-y \sqrt{F}} \operatorname{erfc}\left(\frac{y \sqrt{\operatorname{Pr}}}{2 \sqrt{t}}-\sqrt{\frac{F}{\operatorname{Pr}} t}\right)+e^{y \sqrt{F}} \operatorname{erfc}\left(\frac{y \sqrt{\operatorname{Pr}}}{2 \sqrt{t}}+\sqrt{\frac{F}{\operatorname{Pr}} t}\right)\right\}
\end{gathered}
$$

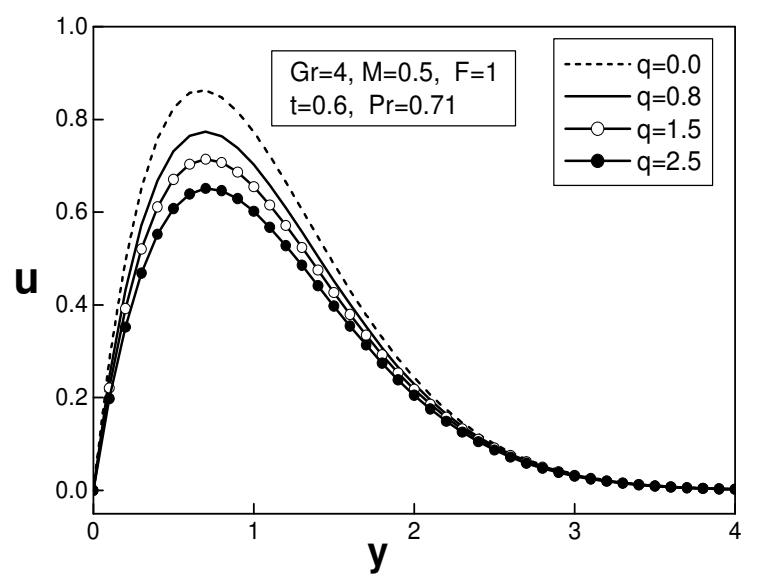

Fig. 3 Effect of $q$ on velocity profile against $y$.

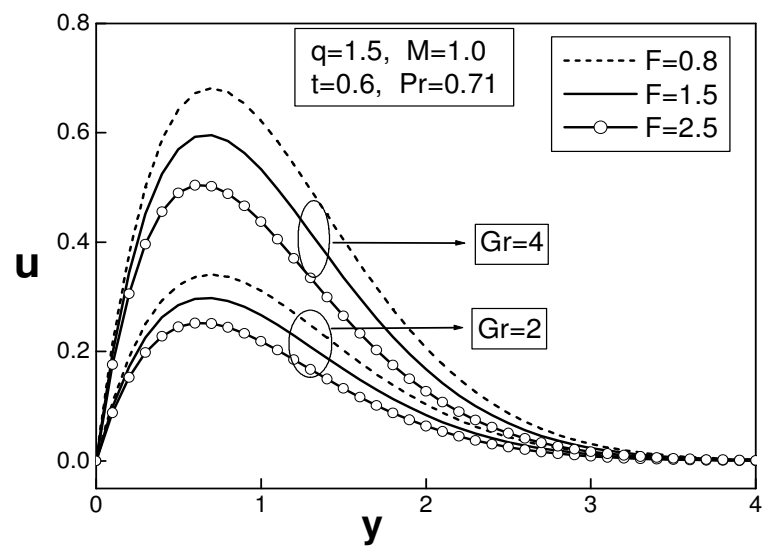

Fig. 4 Effects of $F$ and $G r$ on velocity against $y$.

\subsection{Skin Friction}

The non-dimensional skin-friction $\tau=-\left.\frac{d u}{d y}\right|_{y=0}$ obtained from expression of velocity profile as,

$$
\begin{aligned}
& \tau=\frac{G r}{2(F-M)}[\sqrt{M}\{\operatorname{erfc}(-\sqrt{M t})-\operatorname{erfc}(\sqrt{M t})\} \\
& \left.-\sqrt{F}\left\{\operatorname{erfc}\left(-\sqrt{\frac{F t}{\operatorname{Pr}}}\right)-\operatorname{erfc}\left(\sqrt{\frac{F t}{\operatorname{Pr}}}\right)\right\}\right]+\frac{G r e^{-q t}}{2(F-M-q(\operatorname{Pr}-1))} \\
& {[\sqrt{M-q}\{\operatorname{erfc}(-\sqrt{(M-q) t})-\operatorname{erfc}(\sqrt{(M-q) t})\}} \\
& \left.-\sqrt{F-q \operatorname{Pr}}\left\{\operatorname{erfc}\left(-\sqrt{\frac{(F-q \operatorname{Pr}) t}{\operatorname{Pr}}}\right)-\operatorname{erfc}\left(\sqrt{\frac{(F-q \operatorname{Pr}) t}{\operatorname{Pr}}}\right)\right\}\right]
\end{aligned}
$$




$$
\begin{aligned}
& -\frac{G r}{2}\left\{\frac{1}{F-M}+\frac{1}{F-M-q(\operatorname{Pr}-1)}\right\} e^{-t \frac{F-M}{\operatorname{Pr}-1}} \sqrt{\frac{M \operatorname{Pr}-F}{\operatorname{Pr}-1}} \\
& \left\{\operatorname{erfc}\left(-\sqrt{\frac{(M \operatorname{Pr}-F) t}{\operatorname{Pr}-1}}\right)-\operatorname{erfc}\left(\sqrt{\frac{(M \operatorname{Pr}-F) t}{\operatorname{Pr}-1}}\right)\right. \\
& \left.-\operatorname{erfc}\left(-\sqrt{\frac{(M \operatorname{Pr}-F) t}{(\operatorname{Pr}-1) \operatorname{Pr}}}\right)+\operatorname{erfc}\left(\sqrt{\frac{(M \operatorname{Pr}-F) t}{(\operatorname{Pr}-1) \operatorname{Pr}}}\right)\right\}
\end{aligned}
$$

\subsection{Nusselt number}

The non-dimensional Nusselt number $N u=-\left.\frac{d \theta}{d y}\right|_{y=0}$ obtained from the expression of temperature profile (14) as,

$$
\begin{aligned}
& N u=\frac{2 \sqrt{\operatorname{Pr}}}{\sqrt{\pi t}} e^{-\frac{F t}{\operatorname{Pr}}}+\frac{1}{2} e^{-q t} \sqrt{F-q \operatorname{Pr}}\left\{\operatorname{erfc}\left(-\sqrt{\frac{(F-q \operatorname{Pr}) t}{\operatorname{Pr}}}\right)\right. \\
& \left.-\operatorname{erfc}\left(\sqrt{\frac{(F-q \operatorname{Pr}) t}{\operatorname{Pr}}}\right)\right\}+\frac{\sqrt{F}}{2}\left\{\operatorname{erfc}\left(-\sqrt{\frac{F t}{\operatorname{Pr}}}\right)-\operatorname{erfc}\left(\sqrt{\frac{F t}{\operatorname{Pr}}}\right)\right\}
\end{aligned}
$$

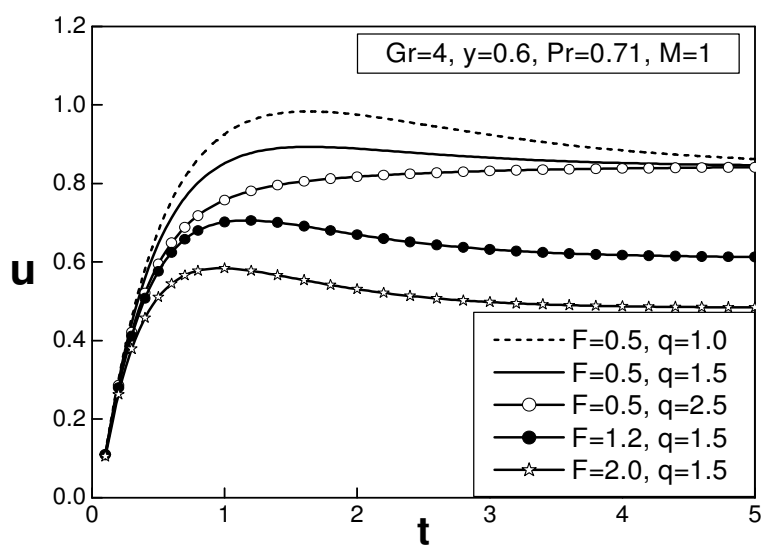

Fig. 5 Effects of $F$ and $q$ on velocity profile against $t$.

\section{RESULTS AND DISCUSSIONS}

In this section we try to grasp the problem physically. To make the physical interpretation of the flow characteristics, figures are plotted for temperature, velocity, Nusselt number and skin friction, showing the effects of different parameters involved in the problem. Fig. 2 shows the effect of transverse magnetic field on velocity against $y$. It is seen that magnitude of velocity goes down as the strength of the transverse magnetic field is increased. Lorentz's force, which grows up as the magnitude of $M$ increases is responsible for this. In fact, Lorentz's force acts as a resisting force on the motion. Velocity profiles against $y$ for different values of $q$ are displayed in Fig. 3. In the figure it is seen that magnitude of velocity gets declined as q increases. A combined effect of Grashof number $(G r)$ and radiation parameter $(F)$ on the flow can be observed from Fig. 4. Grashof number is responsible for the enhancement of buoyancy force. Buoyancy force increases as magnitude of $\mathrm{Gr}$ increases and as such velocity goes up. The same figure shows that magnitude of velocity gets reduced as $F$ increases.

Velocity profile against time under the influences of $F$ and $q$ is displayed in Fig. 5. Observation shows that magnitude of velocity decreases under the effect of both the parameters. In case of $q$, it is interesting to observe that although magnitude of velocity initially suffers a fall for larger value of $q$, as time increases, it is seen that $q$ cannot sustain its effect on the velocity for longer period, as we see in the figure that all the three curves (for $q=1,1.5,2.5$ ) nearly get merged at $t=5$.

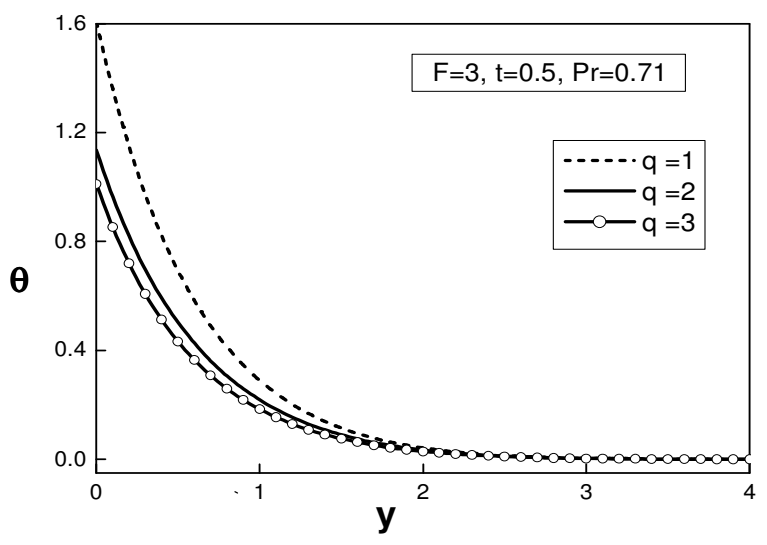

Fig. 6 Effect of $q$ on temperature profile against $y$

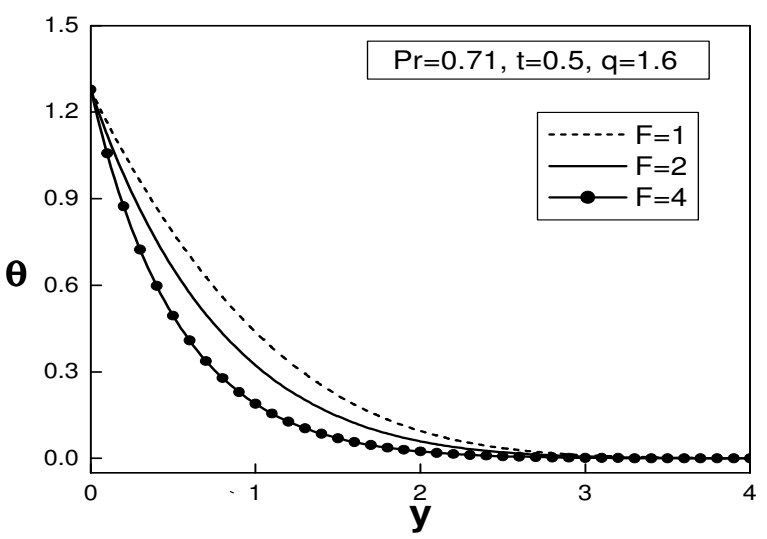

Fig. 7 Effect of radiation on temperature against $y$.

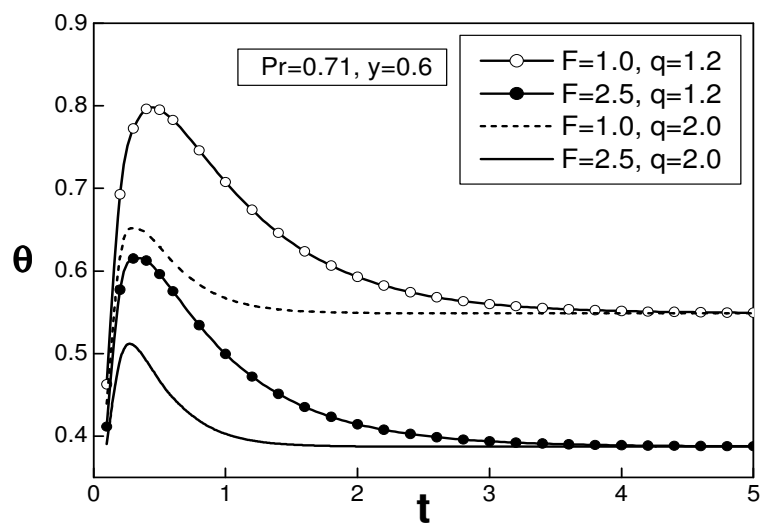

Fig. 8 Effects of $F$ and $q$ on temperature against t.

Temperature profiles versus $y$ for different values of $q$ are demonstrated in Fig. 6 and it is seen that $q$ affects adversely on temperature. Similar effect is also observed in Fig. 7, where effect of radiation $(F)$ on temperature is displayed. Time dependent temperature profile effected by $q$, shown in Fig. 8 indicates that at the vicinity of the plate, effect of $q$ is quite significant, but as time increases the temperature profiles get closer and closer and finally between $t=3$ and $t=4$ each graph attains the ambient temperature. Whereas a different variation is seen in the same figure, when effect of radiation on temperature is considered. It is witnessed that effect of radiation 
remains quite significant till the fluid mass is far away from the plate. Thus variation of radiation creates a long-term effect on temperature.

Skin friction on the plate varying with $F$ and $q$ can be observed in Fig. 9. Skin friction at the plate increases with $q$, of course it becomes steady after some time. Secondly skin friction at the plate significantly grows as $F$ increases. Further Fig. 10 demonstrates that skin friction at the plate is very low in absence of the transverse magnetic field $(M=0)$, but it develops in a drastic manner as soon as the transverse magnetic field is imposed and goes on increasing with increasing values of $M$. Considering the Nusselt number profile for different values of $q$ and $F$, in Fig. 11, observe that Nusselt number (rate of heat transfer) at the plate is enhanced due to the increase of both $q$ and $F$.

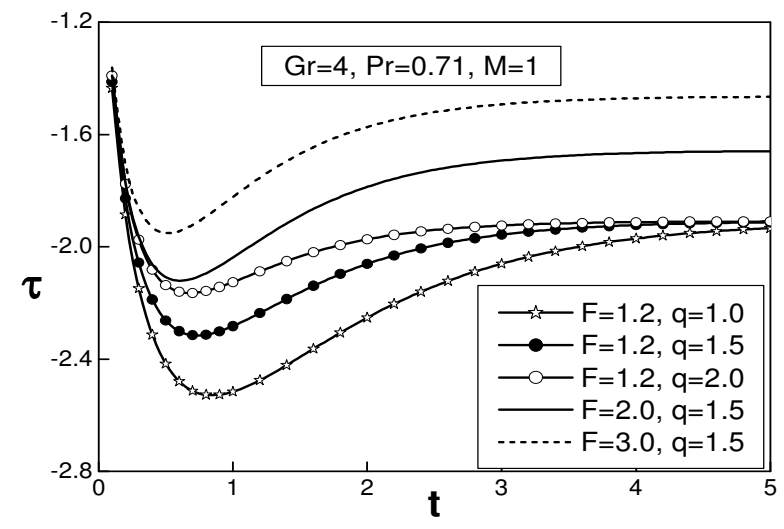

Fig. 9 Skin friction profiles for different values of $F$ and $q$

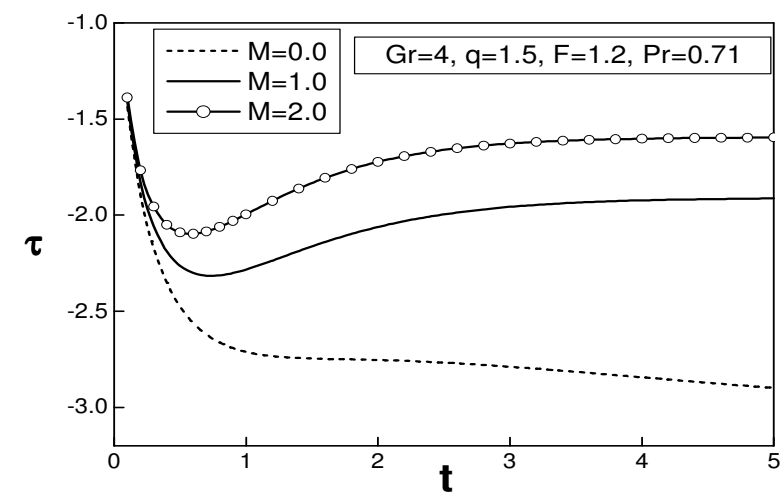

Fig. 10 Effect of magnetic field on skin friction.

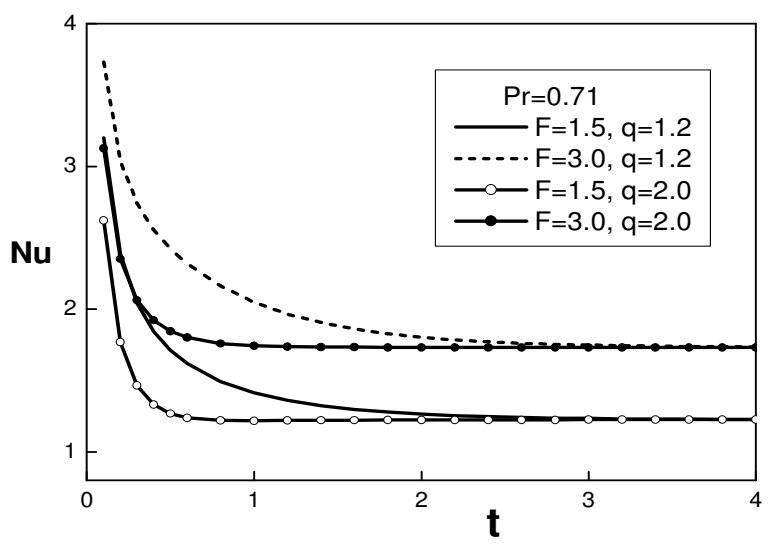

Fig. 11 Variation of Nusselt number profiles with respect to $F$ and $q$

\section{Conclusions}

On the basis of our present study and graphical analysis we summarize the conclusions as follows:

i. $\quad$ Fluid velocity is adversely affected by transversely applied magnetic field as well as radiation.

ii. Temperature of the fluid decreases with the increase in radiation parameter.

iii. Magnitude of skin friction and Nusselt number are enhanced due to the increase in the radiation parameter.

\section{REFERENCES}

Das, U. N., Deka, R. K., and Soundalgekar, V. M., 1996, "Radiation Effects on Flow Past an Impulsively Started Vertical Plate," Journal of Theoretical Mechanics, 1(2), 111-115.

Das, U. N., Deka, R. K., and Soundalgekar, V. M., 1999, "Transient Free Convection Flow Past an Infinite Vertical Plate with Periodic Temperature Variation," Journal of Heat Transfer, 121(4), 1091-1094. http://dx.doi.org/10.1115/1.2826063

Hetnarski, R. B., 1964, "On Inverting the Laplace Transforms Connected with the Error Function," Zastosowania Matematyki, VII, 398-403.

Hetnarski, R. B., 1975, "An Algorithm for Generating Some Inverse Laplace Transforms of Exponential Form," ZAMP, 26(2), 248-253. http://dx.doi.org/10.1007/BF01591514

Hossain, M. A., and Takhar, H. S., 1996, "Radiation Effects on Mixed Convection along a Vertical Plate with Uniform Surface Temperature," Heat and Mass Transfer, 31(4), 243-248. http://dx.doi.org/10.1007/BF02328616.

Ibrahim, F. S., Elaiw, A. M., and Bakr, A. A., 2008, "Influence of Viscous Dissipation and Radiation on Unsteady MHD Mixed Convection Flow of Micro-Polar Fluids," Applied Mathematics \& Information Sciences, 2(2), 143-162.

Muthucumaraswamy, R., and Kumar, S, 2004, "Heat and Mass Transfer Effects on Moving Vertical Plate in the Presence of Thermal Radiation," Theoret. Appl. Mech., 31(1), 35-46. http://dx.doi.org/10.2298/TAM0401035M

Mahmoud Muatafa, A., 2007, "Variable Viscosity Effect of HydroMagnetic Boundary Layer Flow along a Continuously Moving Vertical Plate in Presence of Radiation," Applied Mathematical Sciences, 1 (17), 799-814.

Pal, D., and Chatterjee, S., 2010, "Heat and Mass Transfer in MHD Non-Darcian Flow of a Micro-Polar Fluid over a Stretching Sheet Embedded in a Porous Media with Non-Uniform Heat Source and Thermal Radiation," Communications in Nonlinear Science and Numerical Simulation, 15(7), 1843-1857. doi:10.1016/j.cnsns.2009.07.024

Ramachandra, P. V., and Bhaskar Reddy, N., 2007, "Radiation and Mass Transfer Effect on an Unsteady MHD Free Convective Flow Past a Heated Vertical Plate in Porous Medium with Viscous Dissipation," Theoretical. Applied Mechanics 34(2), 135-160. http://dx.doi.org/10.2298/TAM0702135P 